IFIC-01-37

\title{
Kaluza-Klein theory, AdS/CFT correspondence and black hole entropy
}

\author{
J.M. Izquierdo ${ }^{a 1}$, J. Navarro-Salas ${ }^{b 1}$ and P. Navarro ${ }^{b}$ 也 $^{\text {a }}$ \\ a Departamento de Física Teórica, Universidad de Valladolid \\ E-47011 Valladolid, Spain \\ b Departamento de Física Teórica, Universidad de Valencia \\ and IFIC, Centro Mixto Universidad de Valencia-CSIC \\ E-46100 Burjassot (Valencia), Spain
}

\begin{abstract}
The asymptotic symmetries of the near-horizon geometry of a lifted (near-extremal) Reissner-Nordstrom black hole, obtained by inverting the Kaluza-Klein reduction, explain the deviation of the BekensteinHawking entropy from extremality. We point out the fact that the extra dimension allows us to justify the use of a Virasoro mode decomposition along the time-like boundary of the near-horizon geometry, $\mathrm{AdS}_{2} \times \mathrm{S}^{n}$, of the lower-dimensional (Reissner-Nordstrom) spacetime.
\end{abstract}

\footnotetext{
${ }^{1}$ e-mails: izquierd@fta.uva.es, jnavarro@hal.ific.uv.es, pnavarro@lie.ific.uv.es
} 


\section{Introduction}

The universality of the Bekenstein-Hawking area law of black holes could be explained if the density of the microscopic states is controlled by the conformal symmetry [1]. A nice example of this philosophy is provided by the BTZ black holes [2]. The Bekenstein-Hawking entropy can be derived [3], via Cardy's formula, from the two-dimensional conformal symmetry arising at spatial infinity of three-dimensional gravity with a negative cosmological constant [⿴囗十). Moreover, one can look directly at the black hole horizon and treat it as a boundary. The constraint algebra of surface deformations of the boundary, with additional ad-hoc conditions leads to a Virasoro algebra with a non-vanishing central charge [5, 6, 7]. With the aid of the Cardy formula it is possible to reproduce the adequate density of states leading to the Bekenstein-Hawking area law. Both approaches can be considered simultaneously if one looks at the asymptotic boundary of the near-horizon geometry of black holes. This way one can also explain the entropy of higher -dimensional black holes arising in string theory whose near-horizon geometries are similar to the three-dimensional BTZ black holes.

However, in relevant black holes of general relativity (such as the ReissnerNordstrom solutions) $\mathrm{AdS}_{3}$ does not appear in the near-horizon geometry. It contains, instead, $\mathrm{AdS}_{2}$. The asymptotic symmetries of the $\mathrm{AdS}_{2}$ metric generate a Virasoro algebra [8] with a calculable central charge capable to reproduce the near-extremal black hole entropy [9, 10]. These Virasoro symmetries are those $(t-r)$-diffeomorphisms which leave invariant the large $r$ behaviour of the $\mathrm{AdS}_{2}$ metric. Therefore the Virasoro generators can be regarded as living in the boundary of $\mathrm{AdS}_{2}$, and to perform a mode decomposition one has to make an integration in the time direction. But the canonical formalism requires integration over a space-like slice. So to properly study the $d=2$ case of the AdS/CFT correspondence [11], in terms of asymptotic symmetries, it is necessary to clarify this question. The problem of this extra time-integration also appears in the approach of [7], where it is introduced to properly obtain a central term for a Virasoro algebra. The main aim of this paper is to improve the understanding of this issue: Why do the Virasoro modes in the unconventional time-direction produce the adequate central charge to explain the black hole entropy? We shall focus our attention in the Reissner-Nordstrom (R-N) black holes (in any dimension) and find a natural answer in the context of the Kaluza-Klein (KK) theory.

In Section 2 we shall show how the near-horizon configuration of the 
Reissner-Nordstrom solution can be lifted, inverting the Kaluza-Klein decomposition, to a higher-dimensional metric containing $\mathrm{AdS}_{3}$. In Section 3 we then analyze the $\mathrm{AdS}_{3} / \mathrm{CFT}_{2}$ and $\mathrm{AdS}_{2} / \mathrm{CFT}_{1}$ correspondences within this context, showing that, irrespective of the particular realization of the asymptotic symmetries, the value of the central charge is unique. In Section 4 we argue, from the higher-dimensional point of view, that the mode decomposition in the time direction can be justified. When we add the extra dimension the ordinary time coordinate is converted into a null one in the boundary of the higher-dimensional theory. Therefore one can use Cardy's formula to evaluate, as in [3], the asymptotic growth of states reproducing then the Beckenstein-Hawking formula around extremality. Finally, in Section 5 , we summarize our conclusions.

\section{Relating near-extremal R-N and BTZ black holes by inverting the KK mechanism}

Let us consider a R-N black hole configuration in $(n+2)$ dimensions. The metric field is

$$
d s_{(n+2)}^{2}=-U(r) d t^{2}+\frac{d r^{2}}{U(r)}+r^{2} d \Omega_{(n)}^{2}
$$

where

$$
\begin{gathered}
U(r)=1-\frac{2 G_{n+2} M}{\Gamma_{(n)} r^{n-1}}+\frac{G_{n+2}^{2} Q^{2}}{r^{2(n-1)} \Delta_{(n)}}, \\
\Gamma_{(n)}=\frac{n \nu^{(n)}}{8 \pi}, \quad \Delta_{(n)}=\frac{n}{2(n-1)},
\end{gathered}
$$

$\nu^{(n)}$ is the area of the unit $S^{n}$ sphere,

$$
\nu^{(n)}=\frac{n \pi^{(n+1) / n}}{\Gamma\left(\frac{n+1}{n}\right)}
$$

and the electromagnetic field is given by

$$
A=G_{n+2} \frac{Q}{r^{n-1}} d t
$$

$Q$ is the electric charge and $G_{n+2}$ is the $(n+2)$-dimensional Newton's constant in geometrized units. 
When the R-N configuration is close to extremality and we consider the near-horizon region as follows $(0<\alpha<<1)$

$$
\begin{aligned}
M & =M_{0}\left(1+k \alpha^{2}\right) \\
r & =r_{0}+\alpha x,
\end{aligned}
$$

where $M_{0}$ and $r_{0}$ are the extremal mass and radius

$$
\begin{gathered}
M_{0}=\frac{|Q| \Gamma_{(n)}}{\Delta_{(n)}^{1 / 2}}, \\
r_{0}=\left(\frac{G_{n+2} M_{0}}{\Gamma_{(n)}}\right)^{\frac{1}{n-1}},
\end{gathered}
$$

the leading terms in a series expansion in $\alpha$ of (田) and (5) are

$$
d s_{(n+2)}^{2}=-\left((n-1)^{2} \frac{\alpha^{2} x^{2}}{r_{0}^{2}}-2 \frac{\Delta M}{M_{0}}\right) d t^{2}+\frac{\alpha^{2} d x^{2}}{\frac{(n-1)^{2} \alpha^{2} x^{2}}{r_{0}^{2}}-2 \frac{\Delta M}{M_{0}}}+r_{0}^{2} d \Omega_{(n)}^{2}
$$

where $\Delta M$ is de deviation of the mass from extremality, $\Delta M=M-M_{0}$, and

$$
A=\frac{Q G_{n+2}}{r_{0}^{n-1}}\left(1-(n-1) \frac{\alpha x}{r_{0}}\right) d t .
$$

The metric (10) is just the Robinson-Bertotti geometry $\mathrm{AdS}_{2} \times \mathrm{S}^{n}$, with twodimensional curvature (in the $t-r$ plane) $R^{(2)}=-\frac{2(n-1)^{2}}{r_{0}^{2}}$.

The idea now is to construct a higher-dimensional geometry from the metric and the gauge field by inverting the KK reduction. So the new metric is

$$
d s_{(n+3)}^{2}=d s_{(n+2)}^{2}+\mu^{2}\left(d \theta+Q A_{\mu} d x^{\mu}\right)^{2},
$$

where the KK radius $\mu$ is given by

$$
\mu^{2}=\frac{1}{\Delta_{(n)} Q^{2}}
$$

At leading order in $\alpha$ we have

$$
\begin{aligned}
d s_{(n+3)}^{2} & =-\left(\frac{(n-1)^{2} \alpha^{2} x^{2}}{r_{0}^{2}}-2 \frac{\Delta M}{M_{0}}\right) d t^{2}+\frac{\alpha^{2} d x^{2}}{\frac{(n-1)^{2} \alpha^{2} x^{2}}{r_{0}^{2}}-2 \frac{\Delta M}{M_{0}}}+r_{0}^{2} d \Omega_{(n)}^{2} \\
& +\mu^{2}\left[d \theta+\frac{Q^{2} G_{n+2}}{r_{0}^{n-1}}\left(1-(n-1) \frac{\alpha x}{r_{0}}\right) d t\right]^{2}
\end{aligned}
$$


The relevant fact is that the three-dimensional $(t, r, \theta)$ part of the above metric is just a BTZ black hole with curvature

$$
R^{(3)}=-\frac{2}{l^{2}}
$$

where

$$
l^{2}=\frac{4 r_{0}^{2}}{(n-1)^{2}}
$$

To explicitly relate the three-dimensional sector of (14) with the BTZ black hole we have to perform the natural identification

$$
\theta+\frac{Q^{2}}{r_{0}^{n-1}} G_{n+2} t=x^{+}
$$

together with

$$
2|Q| \Delta_{(n)}^{1 / 2} t=x^{-}
$$

and

$$
\bar{r}^{2}=\frac{(n-1)}{Q^{2} \Delta_{(n)} r_{0}} \alpha x
$$

Plugging these changes of coordinates into (14) we get the following threedimensional metric

$$
d s_{(3)}^{2}=-\bar{r}^{2} d x^{+} d x^{-}+\gamma_{--}\left(d x^{-}\right)^{2}+\gamma_{++}\left(d x^{+}\right)^{2}+\frac{l^{2} \bar{r}^{2} d \bar{r}^{2}}{\bar{r}^{4}-4 \gamma_{++} \gamma_{--}}
$$

where

$$
\begin{aligned}
\gamma_{--} & =\frac{G_{n+2}}{2 r_{0}^{n-1} \Gamma_{(n)} Q^{2} \Delta_{(n)}} \Delta M \\
\gamma_{++} & =\frac{1}{\Delta_{(n)} Q^{2}} .
\end{aligned}
$$

It is interesting to remark that for general chiral functions $\gamma_{++}\left(x^{+}\right), \gamma_{--}\left(x^{-}\right)$ we have the general solution of three-dimensional gravity with a negative cosmological constant $-\frac{2}{l^{2}}$. For constant values of $\gamma_{ \pm \pm}$we have BTZ black holes with

$$
\gamma_{ \pm \pm}=2 G_{3} l\left(M_{B T Z} l \pm J\right)
$$

where $M_{B T Z}$ and $J$ are the corresponding mass and angular momentum. 
If we could establish a one-to-one correspondence between the near-extremal $\mathrm{R}-\mathrm{N}$ and BTZ black holes we could compare the corresponding entropies. There is a straighforward way to do this by comparing the different actions obtained by dimensional reduction. Starting from the $(n+3)$-dimensional action

$$
\frac{1}{16 \pi G_{n+3}} \int d^{n+3} x \sqrt{-g} R
$$

the $\mathrm{KK}$ reduction with constant radius (13) implies that

$$
\frac{1}{G_{n+2}}=\frac{1}{G_{n+3}} 2 \pi \mu
$$

and the factorization of the angular coordinates in the near-horizon region gives

$$
\frac{1}{G_{3}}=\frac{1}{G_{n+3}} \nu^{(n)} r_{0}^{n}
$$

Using the above expressions one finds that

$$
G_{3}=G_{n+2} \frac{2 \pi \mu}{\nu^{(n)} r_{0}^{n}}
$$

The entropy formula for tha BTZ black holes is

$$
S_{B T Z}=\pi \sqrt{\frac{l\left(l M_{B T Z}+J\right)}{2 G_{3}}}+\pi \sqrt{\frac{l\left(l M_{B T Z}-J\right)}{2 G_{3}}}
$$

whereas the formula for R-N black holes is

$$
S_{R N}=\frac{\nu^{(n)} r_{+}^{n}}{4 G_{n+2}}
$$

where $r_{+}$is the outer horizon. Expanding (29) around extremality we find

$$
S_{R N}=\frac{\nu^{(n)} r_{0}^{n}}{4 G_{n+2}}+2 \pi \sqrt{\frac{2 r_{0}^{2} M_{0}}{(n-1)^{2}} \Delta M} .
$$

Comparing (28) and (30) using the relations (21)-(23) and (27) we find agreement only in the extremal configurations $\Delta M=0$ and $M_{B T Z} l=|J|$. We then obtain a result similar to that found in [12]. In this reference it was shown that a $\mathrm{R}-\mathrm{N}$ black hole can be regarded as a dimensional reduction of a 
boosted black string and the near-horizon of the latter contains a BTZ black hole. The entropies of the R-N black hole and the associated BTZ black hole agree at extremality. We have carried out a similar argument by replacing the black string construction by the KK decomposition. However the above mechanisms fail when one compares the entropy deviations from extremality. To properly account for it we shall follow a different route based on the symmetry properties of the near-horizon geometries. This is the main aim of this paper and it will be considered in the next sections.

\section{Asymptotic symmetries and boundary CFT}

The Bekenstein-Hawking area law of BTZ black holes can be understood in terms of the asymptotic symmetries of (20) [3]. The infinitesimal diffeomorphisms $\zeta^{a}$ preserving the asymptotic $r \rightarrow \infty$ form of (20) are

$$
\begin{aligned}
\zeta^{ \pm} & =\varepsilon^{ \pm}\left(x^{ \pm}\right)+\frac{l^{2}}{2 r^{2}} \partial_{\mp}^{2} \varepsilon^{\mp}\left(x^{\mp}\right)+\ldots \\
\zeta^{\bar{r}} & =-\frac{\bar{r}}{2}\left(\partial_{+} \varepsilon^{+}+\partial_{-} \varepsilon^{-}\right)+\ldots
\end{aligned}
$$

where $\varepsilon^{ \pm}\left(x^{ \pm}\right)$are arbitrary chiral functions. Therefore it seems natural to investigate whether these sort of symmetries can also explain the black hole entropy of the near-extremal R-N black holes. The above transformations are asymptotic symmetries of the higher-dimensional near-horizon theory. However only the transformations generated by $\varepsilon^{-}\left(x^{-}\right)$preserve the KK decomposition and can be pushed down. We must note that, in the R-N space-time, the coordinate $x^{-}$is just a time-like coordinate (see (18)), and therefore the transformations generated by $\varepsilon^{-}\left(x^{-}\right)$can be rewritten as

$$
\begin{aligned}
\zeta^{t} & =\varepsilon(t)+\ldots \\
\zeta^{x} & =-x \partial_{t} \varepsilon(t)+\ldots \\
\zeta^{+} & =\frac{l^{2} Q^{2} r_{0}}{2(n-1) \alpha x} \frac{1}{2|Q| \Delta_{(n)}^{1 / 2}} \partial_{t}^{2} \varepsilon(t)+\ldots .
\end{aligned}
$$

The infinitesimal transformations (33), (34) are diffeomorphisms in the R-N spacetime whereas (35) can be interpreted, as usual, as a gauge transforma-

tion. However these transformations do not preserve the asymptotic form of 
the two-dimensional anti-de Sitter metric, although they preserve the asymptotic form of the gauge field. To maintain simultaneously the asymptotic behaviour of the two-dimensional metric and gauge field one can alternatively introduce the following transformations:

$$
\begin{aligned}
\zeta^{t} & =\varepsilon(t)+\frac{l^{4}}{32 x^{2} \alpha^{2}} \varepsilon^{\prime \prime}(t)+\ldots \\
\zeta^{x} & =-x \varepsilon^{\prime}(t)+\ldots \\
\zeta^{+} & =0
\end{aligned}
$$

In contrast with (33)-(35), they are pure diffeomorphisms in the R-N spacetime and the gauge transformation is trivial. We have to remark that these transformations do not preserve the asymptotic form of the three-dimensional anti-De Sitter metric (20). However, both sets of transformations can be used to provide realizations of the AdS/CFT correspondence in a consistent way. In the first case it will be a chiral sector of the $\mathrm{AdS}_{3} / \mathrm{CFT}_{2}$ correspondence, whereas in the second case we shall deal with a sort of $\mathrm{AdS}_{2} / \mathrm{CFT}_{1}$ correspondence. Our task now is to analyze these two sets of symmetries on the spacetime parametrized by $\{t, x\}$ and determine the relevant quantities of the boundary theory required to compute the entropy via Cardy's formula. To this end we shall use the technique introduced in [13], and assume the following asymptotic behaviour of the two-dimensional metric and gauge field

$$
\begin{aligned}
g_{t t} & =-\frac{4}{l^{2}} \alpha^{2} x^{2}+\gamma_{t t}+\ldots \\
g_{t x} & =\frac{\gamma_{t x}}{x^{3}}+\ldots \\
g_{x x} & =\frac{l^{2}}{4 x^{2}}+\frac{\gamma_{x x}}{x^{4}}+\ldots \\
A_{t} & =\frac{Q}{|Q|} \Delta_{(n)}^{1 / 2}\left[1-(n-1)\left(\frac{\alpha x}{r_{0}}-\frac{r_{0}}{2 \alpha x} \gamma_{A_{t}}+\ldots\right)\right] \\
A_{x} & =\frac{\gamma_{A_{x}}}{x^{3}}+\ldots .
\end{aligned}
$$

The action on the radial function, which sometimes is considered as a dilaton, plays no role here. In fact, it could be considered constant. It is just the gauge field which plays a fundamental role. 


\section{1 $\quad \mathrm{AdS}_{3}$-symmetries}

We shall now consider the transformations (33)-(35), which preserve the asymptotic form of the lifted three-dimensional metric. One can show that the unique quantity invariant under the "pure gauge transformations"

$$
\begin{aligned}
\zeta^{t} & =\frac{\alpha^{t}(t)}{x^{2}} \\
\zeta^{x} & =\alpha^{x}(t) \\
\zeta^{+} & =\frac{\alpha^{+}(t)}{x^{2}}
\end{aligned}
$$

is given by

$$
\Theta_{t t}=k_{1}\left(\gamma_{t t}-(n-1)^{2} \gamma_{A_{t}}\right)
$$

where $k_{1}$ is a constant coefficient. Moreover the transformation law of $\Theta_{t t}$ is

$$
\delta_{\varepsilon(t)} \Theta_{t t}=\varepsilon(t) \Theta^{\prime}{ }_{t t}+2 \Theta_{t t} \varepsilon^{\prime}(t)-\frac{k_{1} l^{2}}{2} \varepsilon^{\prime \prime \prime}(t),
$$

which coincides with that of a chiral component of the stress tensor of a twodimensional conformal field theory. The easiest way to determine $k_{1}$, and hence the central charge, is to realize that the value of $\Theta_{t t}$, for a static R-N black hole, should coincide with the mass. More properly, the deviation of the mass from extremality

$$
\left.\Theta_{t t}\right|_{R N}=\Delta M
$$

In order to get (49), one finds that $k_{1}=\frac{M_{0}}{2}$, and therefore the transformation law of $\Theta_{t t}$ is

$$
\delta_{\varepsilon(t)} \Theta_{t t}=\varepsilon(t) \Theta^{\prime}{ }_{t t}+2 \Theta_{t t} \varepsilon^{\prime}(t)-\frac{M_{0} r_{0}^{2}}{(n-1)^{2}} \varepsilon^{\prime \prime \prime}(t)
$$

\section{2 $\quad \mathrm{AdS}_{2}$-symmetries}

Alternatively we can consider the symmetries (36-38), which preserve the asymptotic form of the two-dimensional metric. In this case the invariant quantity under the "pure gauge diffeomorphisms"

$$
\begin{aligned}
\zeta^{t} & =\frac{\alpha^{t}(t)}{x^{4}}+\ldots \\
\zeta^{x} & =\frac{\alpha^{x}(t)}{x}+\ldots,
\end{aligned}
$$


is

$$
\Theta_{t t}=k_{2}\left(\gamma_{t t}-\frac{8}{l^{2}} \gamma_{x x}\right)
$$

where $k_{2}$ is again a constant coefficient. The transformation law of $\Theta_{t t}$ is

$$
\delta_{\varepsilon(t)} \Theta_{t t}=\varepsilon(t) \Theta^{\prime}{ }_{t t}+2 \Theta_{t t} \varepsilon^{\prime}(t)-\frac{k_{2} l^{2}}{4} \varepsilon^{\prime \prime \prime}(t)
$$

The value of $k_{2}$ can be determined immediately by requiring

$$
\left.\Theta_{t t}\right|_{R N}=\Delta M
$$

We find that

$$
k_{2}=M_{0}
$$

and this reproduces the same transformation law as that of (50).

\section{The role of the extra dimension and black hole entropy}

One of the effects of introducing the extra Kaluza-Klein coordinate is that it converts the near-horizon geometry $\mathrm{AdS}_{2} \times \mathrm{S}^{n}$ (10) into the $\mathrm{AdS}_{3} \times \mathrm{S}^{n}$ geometry. This has important consequencies for the causal structure of the boundaries. For $\mathrm{AdS}_{2}$ the boundary consists of two disconnected time-like real lines. It can be embedded (more precisely, the connected part corresponding to $x=+\infty$ ) into the cylindrical boundary of $\mathrm{AdS}_{3}$ through the relations (17)-(18). The lifted time-like boundary of $\mathrm{AdS}_{2}$ becomes a null slice of the $\mathrm{AdS}_{3}$ boundary and then one can perform the usual Fourier mode decomposition of $\Theta_{t t}(t)$, which now bcomes $\Theta_{--}\left(x^{-}\right)$, as follows

$$
L_{n}^{R}=\frac{2 Q \Delta_{(n)}^{1 / 2}}{2 \pi} \int_{0}^{2 \pi} d x^{-} \Theta_{--}\left(x^{-}\right) e^{i n x^{-}} .
$$

Using either (50) or (54) (with 56) we get a Virasoro algebra $\left(\varepsilon_{m}=e^{i m x^{-}}\right)$

$$
\left\{L_{n}^{R}, L_{m}^{R}\right\}=\delta_{\varepsilon_{m}} L_{n}^{R}=(n-m) L_{n+m}^{R}+\frac{c}{12} n^{3} \delta_{n,-m}
$$

with a central charge

$$
c=\frac{24 M_{0} r_{0}^{2} Q \Delta_{(n)}^{1 / 2}}{(n-1)^{2}}
$$


Note that for the $(n+2)$-dimensional theory (57) involves a time integration which is not permitted by the canonical formalism, only in the higherdimensional theory is that step allowed. Moreover the quantities (57) are conserved charges (i.e., time independent). In contrast, in the $(n+2)$ dimensional space-time only the zero mode of $\Theta_{t t}$ is a well-defined conserved charge. This reinforces the idea that a higher-dimensional perspective is necessary to properly define a Virasoro algebra leading, via Cardy's formula

$$
\log \rho \sim 2 \pi \sqrt{\frac{c L_{0}^{R}}{6}}
$$

to the adequate density of states. The $L_{0}^{R}$-Virasoro charge takes the value (for static R-N black holes)

$$
L_{0}^{R}=\frac{\Delta M}{2 Q \Delta_{(n)}^{1 / 2}}
$$

and together with (59) we obtain

$$
\Delta S=2 \pi \sqrt{\frac{2 r_{0}^{2} M_{0}}{(n-1)^{2}} \Delta M}
$$

in exact agreement with the entropy deviation from extremality of R-N black holes.

\section{Conclusions and final comments}

The search for a universal mechanism responsible for black hole entropy involves two basic ingredients: near-horizon symmetries and conformal field theory. For R-N black holes the near-horizon geometry contains $\mathrm{AdS}_{2}$ and the corresponding conformal generators live in the time-like boundary of $\mathrm{AdS}_{2}$ thus making problematic the role of the Virasoro modes (in a time direction) in classifying states. In the analogous situation in $\mathrm{AdS}_{3}$, the space-like slice of the boundary is a circle. The relevant diffeomorphisms are parametrized by two functions which translate into two Virasoro algebras. The Virasoro generators are the modes on the space-like circle. Moreover, the holomorphicity of the chiral components of the stress tensor implies that one can convert the integral over a spatial coordinate into an integral over a null 
coordinate. Hence, it can be concluded that the Hilbert space on this circle has a growth of states given by Cardy's formula. In this paper we have shown that the extra Kaluza-Klein dimension allows to embed the time-like boundary of $\mathrm{AdS}_{2}$ into a null slice of $\mathrm{AdS}_{3}$ providing consistency to the use of Cardy's formula to evaluate the entropy deviation from extremality in a statistical way.

Acknowledgements. P. Navarro acknowledges the Ministerio de Educación y Cultura for a FPU fellowship. This work has been partially supported by the DGICYT (Spain), and by the Junta de Castilla y León (J.M.I.).

\section{References}

[1] S. Carlip, Nucl. Phys. Proc. Suppl. 88 (2000) 10

[2] M. Bañados, C. Teitelboim and J. Zanelli, Phys. Rev. lett. 69 (1992) 1849

[3] A. Strominger, JHEP 02 (1998) 009

[4] J.D. Brown and M. Henneaux, Commun. Math. Phys. 104 (1986) 207

[5] S.N. Solodukhin, Phys. Lett. B454 (1999) 213

[6] S. Carlip, Phys. Rev. Lett. 82 (1999) 2828

[7] S. Carlip, Class. Quant. Gravity 16 (1999) 3327

[8] M. Cadoni and S. Mignemi, Phys. Rev D59 (1999) 081501; Nucl. Phys. B557 (1999) 165

[9] J. Navarro-Salas and P. Navarro, Nucl Phys. B579 (2000) 250

[10] D.J. Navarro, J. Navarro-Salas and P. Navarro, Nucl. Phys. B (2000)

[11] O. Aharony, S.S. Guber, J.M. Maldacena, H. Ooguri and Y. Oz, Phys. Rept. 323 (2000) 183

[12] Y. Satoh, Phys. Rev. D59 (1999) 084010

[13] J. Navarro-Salas and P. Navarro, Phys. Lett. B439 (1998) 262 